\title{
La problématique action research/recherche-action et le travail coopératif
}

Jean-Paul Narcy-Combes

\section{(2) OpenEdition}

\section{Journals}

Édition électronique

URL : http://journals.openedition.org/asp/2835

DOI : 10.4000/asp.2835

ISSN : 2108-6354

\section{Éditeur}

Groupe d'étude et de recherche en anglais de spécialité

\section{Édition imprimée}

Date de publication : 1 décembre 1998

Pagination : 229-238

ISSN : 1246-8185

\section{Référence électronique}

Jean-Paul Narcy-Combes, « La problématique action research/recherche-action et le travail

coopératif », ASp [En ligne], 19-22 | 1998, mis en ligne le 16 mars 2012, consulté le 19 avril 2019. URL http://journals.openedition.org/asp/2835 ; DOI : 10.4000/asp.2835

Ce document a été généré automatiquement le 19 avril 2019

Tous droits réservés 


\title{
La problématique action research/ recherche-action et le travail coopératif
}

\author{
Jean-Paul Narcy-Combes
}

\section{Introduction}

1 Décrire des comportements idéalisés ne signifie pas que l'on sait les mettre en pratique ${ }^{1}$. Pour tenter de clarifier la question, je développerai ma réflexion en tentant de garder constamment à l'esprit les données théoriques et les développements pratiques.

\section{Action research : une dimension idéologique}

Le concept d'action research remonte aux années 1970. Il est dans la mouvance de l'esprit libertaire de l'époque, mais est d'origine britannique. Il s'agit d'une recherche qui se veut positive, orientée vers l'action afin de résoudre des problèmes de terrain. On la décrit: réflexive, démocratique et favorisant un développement humaniste.

3 Il y a clairement un parti pris idéologique qui explique que certains chercheurs aient fait preuve de réserve à son égard.

Trois types d'action research ont été décrits (Ellis 1997) :

- «technique » (découverte du savoir, la terminologie est celle d'Ellis),

- recherche pédagogique (contrôle des pratiques),

- critique (permettant la « libération » de l'enseignant du poids des concours, de l'autorité, de la tradition et de ses propres représentations).

4 On retrouve dans le troisième type la dimension idéologique qui a déjà été soulignée et sur laquelle nous reviendrons.

$5 \quad$ Action research est présentée comme ascendante (bottom up), elle part du terrain. Dans l'ensemble, les humains et donc les enseignants rejettent la réflexion critique, qui, si elle 
remet le monde en cause, les remet également en cause... et action research n'a pas connu le développement que ses fondateurs espéraient, en France du moins.

\section{Tentative de définition et caractéristiques}

\section{Définition}

6 Comme nous l'avons vu, il s'agit d'une recherche liée à la réalisation pratique de résolutions de problèmes de terrain où la réflexion a une dimension aussi bien pratique que critique. Cette définition justifie les spécificités générales de ce type de recherche.

\section{Spécificités générales}

Tableau 1. Spécificités générales

\begin{tabular}{|c|c|}
\hline $\begin{array}{l}\text { Les enseignants } \\
\text { - se soumettent et soumettent leur pratique à une } \\
\text { observation critique } \\
\text { - tentent de lier des idées et des observations } \\
\text { empiriques } \\
\text { - tentent d'exploiter ce processus pour eux-mêmes et } \\
\text { les autres, par écrit } \\
\text { - veulent améliorer leur(s) pratique(s), dans une } \\
\text { situation spécifique du point de vue de leur priorité et } \\
\text { de leur préoccupation } \\
\text { - veulent aller de l'avant }\end{array}$ & $\begin{array}{l}\text { Fonctionnement pratique } \\
\text { - la recherche ne doit ni gêner, ni } \\
\text { interrompre le travail initial de } \\
\text { l'enseignant } \\
\text { - le recueil des données doit être facile } \\
\text { - la méthodologie de recherche doit } \\
\text { s'adapter au contexte } \\
\text { - l'enseignant doit s'engager } \\
\text { personnellement } \\
\text { - des précautions éthiques sont } \\
\text { impératives (confidentialité) }\end{array}$ \\
\hline
\end{tabular}

\section{Conséquences pratiques}

7 La RA/AR est exigeante et requiert une réflexion préalable minutieuse sur sa faisabilité.

8 Elle est de courte durée (moins de trois ans), mais exige beaucoup de temps d'implication pour le(s) chercheur(s).

9 Comme elle est dynamique, elle doit rester simple dans son déroulement (alors même que son organisation est rigoureuse) et ceci peut poser des problèmes au niveau institutionnel. des participants.

Elle impose un monitoring, un suivi rigoureux, qui, par contre, ne saurait menacer personne.

Elle requiert une grande connaissance de soi (cf. la notion d'auto-analyse du chercheur d'E. Morin, entre autres, in Narcy 1998).

Il arrive que son étendue, et ses ambitions soient trop grandes, car le problème de terrain n'a pu être décomposé de façon satisfaisante.

Rendre compte est parfois difficile, selon le «niveau scientifique » des participants, mais est impératif.

Sans compte rendu, on reste souvent au niveau des pratiques innovantes qui constituent, en quelque sorte un sous-ensemble de la recherche-action. 


\section{Un sous-ensemble : l'innovation}

L'innovation ne fait pas, comme nous l'avons vu, l'objet d'un compte rendu scientifique. Cela n'est pas sans un effet en amont.

Les fondements de l'innovation sont le plus souvent issus de représentations culturelles ou personnelles que des résultats des recherches dans les domaines concernés.

Les facteurs impliqués sont:

- le contexte socioculturel,

- la personnalité, les compétences des participants,

- la méthode de mise en place (ascendante, issue du terrain, ou descendante, décidée par les autorités),

- les caractéristiques du projet, en particulier les objectifs apparents et les objectifs inconscients (hidden agenda).

Dans une action innovante, ces facteurs ne sont pas abordés explicitement: on reste souvent dans le domaine des motivations profondes (Narcy 1998) et des jeux d'influence.

\section{Les objectifs de la recherche-action}

Ces objectifs sont contradictoires :

- Faire des recherches sur une situation ce qui implique une distanciation (Narcy 1998).

- Agir sur cette situation, ce qui au contraire se justifie par un engagement.

Ces objectifs sont également locaux (liés à un contexte déterminé) et pédagogiques (liés à une pratique ; plus que généraux et didactiques).

Néanmoins ils sont justifiés, en effet la RA peut générer des/une théorie(s) en apportant des "preuves" pratiques plus que statistiques (il n'est pas toujours facile d'avoir des preuves dont la validité statistique est totalement probante) même en recherche non action.

23 De plus, il est nécessaire de faire des études expérimentales sur le terrain, de théoriser les pratiques, et de combler un fossé entre connaissance scientifique et connaissance pratique (pourquoi une action a réussi sans correspondre à une théorie).

De ce fait, la RA permettra d'améliorer l'action (les pratiques) en soulevant les problèmes, les causes de préoccupations, tels que les praticiens les perçoivent.

L'auto-analyse des participants permet de percevoir l'influence des motivations profondes sur les représentations et les pratiques et favorise la modification des stratégies d'action sans menacer quiconque. Un regard critique, libéré des représentations personnelles, mais également des «diktats » de l'autorité, favorisera l'efficacité des pratiques.

\section{Les domaines d'application}

\section{En général}

- dynamisation pour l'action (distribution des responsabilités pour que chacun se sente motivé), 
- activités individuelles, relations humaines, amélioration du moral,

- analyse d'emplois (détermination des caractéristiques des postes et redéfinition),

- changements institutionnels,

- planification, mise en place d'actions,

- innovation,

- résolution de problème,

- apport théorique à valider.

\section{Dans l'enseignement}

- remédiation/amélioration des pratiques : méthodes d'enseignement/apprentissage, mise en place de nouvelles organisations, stratégies d'apprentissage, procédures d'évaluation,

- études d'attitudes et de valeurs et modifications des comportements (travail d'équipe)

- formation continue (savoir et regard critique)

- innovation (avec compte rendu, et donc organisation et suivi rigoureux)

- amélioration du lien terrain / recherche (équipes mixtes, chercheurs / praticiens)

- amélioration des méthodes intuitives de théorisation de la pratique : création d'un lien entre chercheurs et praticiens, apport de rigueur dans la résolution de problèmes.

\section{Cycle de la recherche-action}

Le diagramme à la figure 1 illustre la complémentarité des deux termes de l'appellation. C'est la recherche qui nourrit l'action. C'est au niveau de la rédaction que l'action nourrira la recherche.

Figure1.

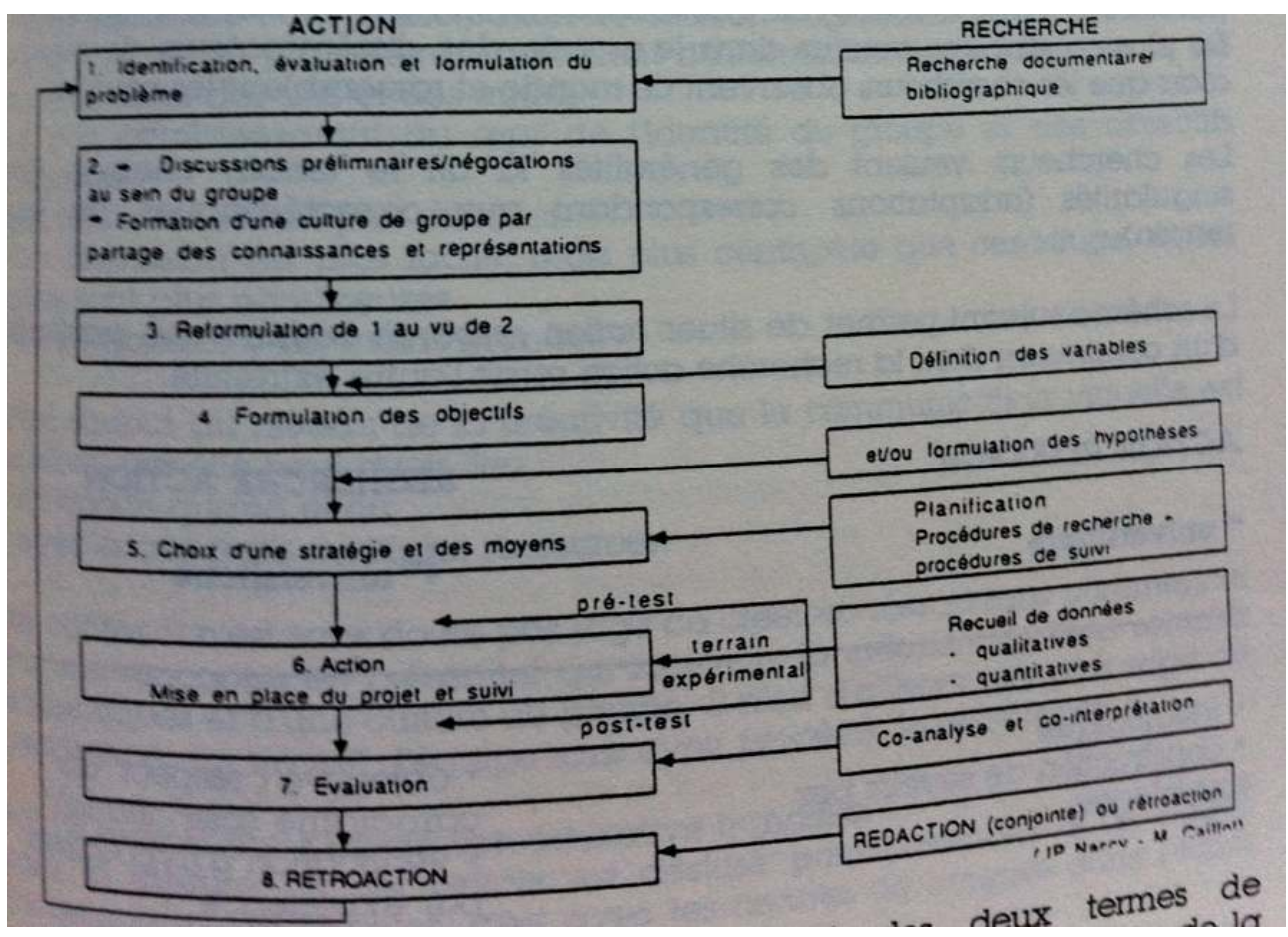

La notion de culture commune est importante. C'est elle qui permettra une authentique complémentarité, mais qui facilitera également l'intercompréhension entre des participants parfois fort différents. Cette culture commune ira des théories scientifiques 
(mises à la portée de tous) à la connaissance des pratiques de chacun et des rouages du système.

La rétroaction sera permanente. C'est ce qui explique les difficultés qui peuvent surgir en milieu institutionnel quand on décèle une résistance dont la levée risquerait une profonde réorganisation.

En termes de recherche, les critères sont les mêmes que dans les autres formes de recherche (cf. Narcy 1998 ; Seliger \& Shohamy 1989).

\section{Pourquoi y a-t-il un dilemme?}

La recherche traditionnelle est déroutante, «jargonante ». Elle est peu orientée vers la pratique. Théorique donc, elle est difficile à réfuter. De plus, les chercheurs défendent jalousement un terrain que les enseignants n'ont pas nécessairement envie d'envahir par manque d'habitude, mais surtout, et à tort, de confiance.

31 Pourtant Widdowson, cité par Ellis, affirme que la théorisation doit être centrée sur le « client », dans ce cas sur le praticien.

Chercheurs et praticiens ont des valeurs et des objectifs différents, tout comme les institutions d'enseignement. De ce fait, les praticiens parviennent mal à concevoir que leur recherche peut avoir une légitimité. De plus ce sont des acteurs dans le monde, des disséminateurs de savoir, alors que les chercheurs observent ce monde et rassemblent les savoirs.

33 Les chercheurs veulent des généralités là où le terrain cherche des singularités (adaptations correspondant aux caractéristiques de leur terrain).

Le schéma à la figure 2 permet de situer action research comme une extrémité d'un continuum dont la recherche-action serait l'autre extrémité.

Figure 2

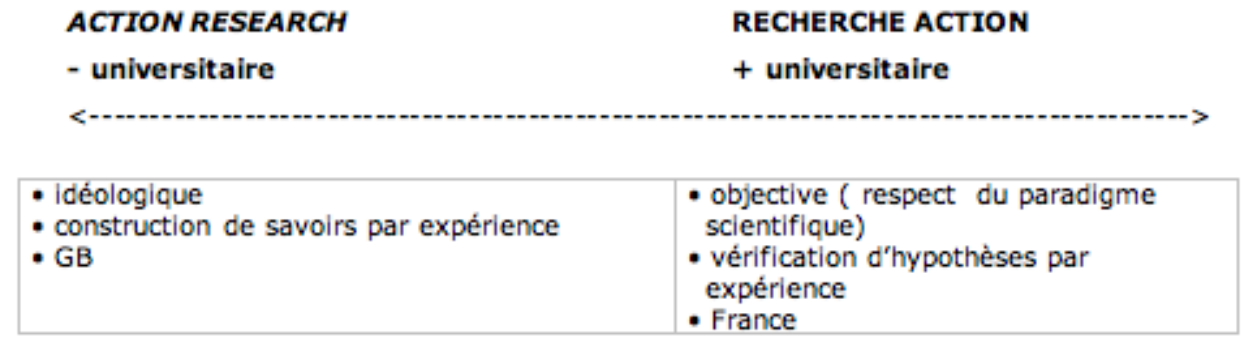

Chaque action qui se réfère à cette double appellation peut se situer sur un des points du continuum. La validité du résultat sera plus pratique du côté d'action research, et plus théorique de l'autre, et ce ne sont pas là des critères de respectabilité.

Il n'est pas moins vrai qu'en France, les recherches de type action research n'ont pas nécessairement la reconnaissance voulue. Sans doute parce qu'il importe alors qu'un chercheur, ou qu'une équipe de chercheurs, situe les résultats dans la problématique du domaine, en soulignant en quoi ils contribuent à valider ou augmenter les savoirs. 


\section{Les réseaux, la RA et le travail coopératif}

\section{La recherche-action et la notion de réseau}

Cette notion est constitutive de la recherche action dès les années 1970 par le fait que les données et les résultats devaient circuler, la notion de culture de groupe et le fait que connaissances et pratiques devaient être améliorées par échange.

Le concept de qualité du temps d'échange dans les réseaux a été mis en relief dès les débuts (comment bien gérer ce temps d'échange pour qu'il ne pèse pas trop).

Caractéristiques de la communication assistée par ordinateur (Worschauer in Darleguy et al. 1997)

Dans la mesure où les réseaux sont appelés à tous être sur support électronique, il importe de connaître comment le support influe.

Selon les études que cite Worschaurer le média est «démocratique» plus égalitaire et la participation de chacun augmente ; en revanche, les partenaires manquent d'information sur le contexte et les traits sociaux des uns et des autres; il y a affaiblissement du sens de l'identité du groupe et des objectifs communs (les décisions seront plus difficiles); la discussion est plus facile, mais plus centripète que centrifuge, et les idées sont plus nombreuses; la conformité y est moins grande.

41 C'est surtout au niveau de la créativité que la communication virtuelle est positive, car elle peut avoir lieu n'importe quand et où, avec accès à de multiples ressources.

Il n'est sans doute pas sage de créer un réseau sans commencer par une rencontre en présentiel qui facilitera la création de liens sociaux authentiques et d'une culture de groupe. Il n'est d'ailleurs pas plus sage de commencer un travail d'équipe sans avoir procédé de cette façon.

\section{Le réseau dans l'institution éducative française}

Dans la mesure où cet article est destiné principalement à un public d'enseignants de langues, c'est avec les centres de langues dans l'esprit que ces lignes sont rédigées.

L'expérience montre que les rôles doivent être bien délimités et l'expertise de chacun bien définie.

L'administrateur : il doit être à l'aise en TIC (Technologie de l'information et de la communication) et en didactique des langues. Son statut doit lui permettre de dialoguer sans difficulté avec ses partenaires dans l'institution.

Le médiatiseur : il semble préférable que ce soit un informaticien créatif, plutôt qu'un enseignant. Souvent des enseignants se "lancent» dans la médiatisation, gare à l'amateurisme, mais surtout à la surcharge de travail.

Le documentaliste : aidera l'équipe à gérer les documents.

Les techniciens : certaines tâches, téléchargement, numérisation, etc., sont purement techniques et il semble dommageable que les enseignants les accomplissent.

Didacticiens et pédagogues : s'il y a création de tâches, il semble préférable qu'il existe une expertise didactique dans l'équipe. Autrement, on risque de mettre de nouveaux habits sur des pratiques démodées.

50 Le(s) tuteur(s) : peut-on s'improviser tuteur? Il y a là le sujet d'une intéressante recherche-action (cf. Narcy in Darleguy et alii 1997). 


\section{Le contexte français et le travail d'équipe} en réseau, la complémentarité des résultats importe autant que la hiérarchie des actions. Il est parfois étonnant que dans un pays aux idéaux si foncièrement égalitaires, il reste des zones où les hiérarchies demeurent avec des fondements dont la légitimité scientifique, dans des domaines complexes, reste à prouver.

\section{BIBLIOGRAPHIE}

Andersen, R. 1983. Pidginization and Creolization as Language Acquisition. Rowley, MA : Newbury House.

Annoot, E. 1996. Les formateurs aux nouvelles technologies : le sens du changement. Paris : Ophrys.

Brown, J. D. 1988. Understanding Research in Second Language Learning. Cambridge : Cambridge University Press.

Buser, P. 1998. Cerveau de soi, cerveau de l'autre. Paris : Odile Jacob.

Cohen, L. et L. Manion. 1988. Research Methods in Education. Londres : Routledge.

Cyrulnik, B. 1983. Mémoire de singe et parole d'homme. Paris : Hachette.

Cyrulnik, B. 1997. L'ensorcellement du monde. Paris : Odile Jacob. 
Darleguy, V. et alii (dir.). 1997. Les nouvelles technologies éducatives dans l'apprentissage des langues vivantes : réflexion théorique et applications pratiques. Lyon : INSA.

Ellis, R. 1997. SLA Research and Language Teaching. Oxford :Oxford University Press.

Gardner, H.1996. Les intelligences multiples. Lyon : Retz.

Gravitz, M. 1991. Méthodes des sciences sociales, 8e édition. Paris : Dalloz.

Hustler, D. et alii. 1986. Action Research in Classrooms and Schools. Londres : Allen and Unwin.

Laborit, H. 1994. La légende des comportements. Paris : Flammarion.

Larsen-Freeman, D. et M.H. Long. 1991. An Introduction to Second Language Research. London \& New York : Longman.

Linard, M. 1996. Des machines et des hommes, éd. révisée. Paris : L'Harmattan.

Little, D. 1997. « Strategies, counselling and culluide difference: why we need an anthropological understanding of learner autonomy? ». In Proceedings, Sixth Conference in Developing Autonomous Learning Barcelona, 3-6 Septembre 1997.

Loubet del Bayle, M. 1986. Introduction aux méthodes des sciences sociales. Paris : Privat.

Macé, G. 1991. Guide d'élaboration d'un projet de recherche. Paris : Éditions Universitaires.

Narcy, J.-P. 1998. « Représentations, apprentissages et supports multimédias ». Cahiers de l'APLIUT $17 / 3,14-24$.

Narcy, J.-P. 1997. « Vers une pratique théorisée et humaniste . In Ginet, A., Du laboratoire de langues à la salle de cours multimédias. Paris : Nathan.

Pateau, J. 1994. «Approche comparative interculturelle : étude d'entreprises françaises et allemandes ». Thèse de doctorat, Université de Paris 10 Nanterre.

Quivy, R. et L. Van Campenhoudt. 1988. Manuel de recherches en sciences sociales. Paris : Dunod.

Schumann, J. 1978. « Social and psychological factors in second language acquisition ». In Richards, J. (dir.), Understanding Second and Foreign Language Learning. Rowley, MA : Newbury House

Seliger, H.W. et E. Shohamy. 1989. Second Language Research Methods. Oxford : Oxford University Press.

Skehan, P. 1997. A Cognitive Approach to Second Language Learning. Cambridge : Cambridge University Press.

Sturge-Moore, O. 1997. « De la nécessaire inclusion du contexte culturel dans les cours d'anglais de spécialité ». Thèse de doctorat, Université de Bordeaux 2.

Varela, F. J. 1996. Quel savoir pour l'éthique? trad. de l'anglais. Paris : Éditions de la découverte.

\section{NOTES}

1. Ce texte reprend et développe une communication faite au colloque organisé à l'Université Joseph Fourier de Grenoble en septembre 1998. 


\section{RÉSUMÉS}

L'insertion institutionnelle d'actions innovantes confronte les chercheurs et les praticiens à un équilibre délicat entre la réflexion théorique dans un cadre méthodologique rigoureux et les aléas de la pratique. La recherche-action est une réponse à cet équilibre. Cette réponse sera d'autant plus efficace si l'on perçoit que toute recherche-action se situe sur un continuum entre deux extrémités, l'une plus française dans ses principes, l'autre plus anglo-saxonne. À une époque où la notion de réseau nous confronte à la gestion d'un travail en équipe "virtuelle ", la recherche-action nous permet de résoudre en souplesse, mais avec rigueur, nombre de problèmes, en prenant en compte les paramètres socio-affectifs des phénomènes que l'on veut gérer.

With the constant introduction of new and innovative techniques, researcher and teacher alike must strike a delicate balance between considering theory in a strict methodological context and the uncertainties arising in practice. Research action is an attempt to respond to this need for balance. It is all the more effective if seen in the context of a continuum between two extremes, one more French in its principles and the other tending towards Anglo-Saxon principles. Now that networks provide us with the opportunity to work in a "virtual" team, research action gives us both the flexibility and precision to resolve a number of problems by considering the socioaffective aspects of the phenomena being dealt with.

\section{INDEX}

Keywords : cultural feature, epistemology, innovation, methodology, network, action research, team

Mots-clés : épistémologie, équipe, innovation, méthodologie, recherche-action, réseau, spécificité culturelle

\section{AUTEUR}

\section{JEAN-PAUL NARCY-COMBES}

Jean-Paul Narcy-Combes est professeur à l'Université de Technologie de Compiègne. Il fait partie de l'équipe nationale de pilotage du DEA d'anglais de spécialité. Ses recherches actuelles se centrent principalement sur les problèmes épistémologiques et méthodologiques qu'implique la complexité de l'apprentissage des langues à l'heure où les TIC apportent des réponses nouvelles. Ses dernières publications comprennent en particulier : chapitre 2 de Ginet, Alain, Du laboratoire de langues à la salle de cours multi-médias (Nathan, 1997) ; « Représentation, apprentissage et supports multi-médias » (Cahiers de l'APLIUT 17/3, 1998) ; « Le Monde à notre portée, d'un regard ethnocentré à une intelligence avertie » (Études de Linguistique Appliquée 112, 1998). jean-

paul.narcy-combes@wanadoo.fr 